\title{
Digestive Surgical Emergencies at Analankininina Toamasina Madagascar University Hospital during the Covid-19 Pandemic
}

Razafindraibe $\mathrm{VB}^{1}$, Rasataharifetra ${ }^{1}$, Rahantasoa $\mathrm{FCFP}^{2}$, Samison $\mathrm{LH}^{2}$, Rakotoarijaona $\mathrm{AH}^{1}$ rcasimirfleurprudence@gmail.com

${ }^{1}$ Visceral surgery department of the Analankinina University Hospital Center, Madagascar

${ }^{2}$ Visceral surgery department of the Joseph Ravoahangy Andrianavalona University Hospital Center, Madagascar

Received: September 1, $2020 \quad$ Received in Revised: September 8, $2020 \quad$ Accepted: September 10, 2020

\begin{abstract}
During the period of total containment during the Covid-19 pandemic; our department became the sole treatment center for all surgical emergencies. The influx of many patients made the task very difficult for the surgeon. This is a 3-month descriptive prospective study in the visceral surgery department at the Analankininina Toamasina University Hospital Center. Of the 81 patients hospitalized during the study, 32 patients were admitted for digestive surgery emergency, i.e. $39.50 \%$. The mean age was 34.87 years with a sex ratio of 1.66 , predominantly male. Abdominal pain occupied $43.75 \%$ of the reason for consultation, the most frequent pathologies were acute intestinal obstruction (18.75\%) and acute appendicitis (18.75\%). An exploratory laparotomy is performed on $40.62 \%$ of patients. Many patients were operated on for proctologic emergency (18.75\%). The average length of hospitalization is 4.18 days. The visceral emergencies are present despite the presence of the Covid-19 pandemic and the activities of the health personnel are uninterrupted.
\end{abstract}

Keywords: Surgery; Covid-19; Acute Intestinal Occlusion; Pandemic; Visceral Emergency

\section{Introduction}

The coronavirus epidemic of 2019 (COVID-19) was declared a "global public health emergency" by the World Health Organization (WHO) on January 30, 2020 and was declared a pandemic on March 11, 2020 (Berthé, 2008). It now affects the entire population and the entire health system. Since the appearance of the new Covid-19 pandemic, patient care has been modified in hospitals in our region. Some surgical centers have been closed to receive only patients infected with SARS-CoV-2. Due to the existence of the pandemic, patients with surgical emergencies are all being cared for in our hospital.

We report on the work of healthcare workers in the visceral surgery department during the pandemic to show the impact of the pandemic on digestive surgical emergencies.

\section{Methods}

Our study was prospective and descriptive over a period of 3 months from March 2020 to May 2020. We included all patients operated on for visceral emergency during the Covid19 containment period. The study is taking place in the visceral surgery department of the Analankininina Toamasina University Hospital Center, Madagascar. Thegae sample was 
exhaustive. The variables studied included; age, gender, surgical indications, treatment, evolution.

\section{Results and Discussion}

We had a large number of patients in the visceral surgery department during containment, $\mathrm{n}=81$. There were: nineteen (19) traumas of the musculoskeletal system, 13 neurosurgical pathologies, 15 urological pathologies. Visceral surgical emergencies represented $39.50 \%$ or $n=32$ during the study period. The mean age was 34.87 years [95\% CI: 27.3243-45.9138]. The sex ratio was predominantly male giving a sex ratio of 1.66. Exploratory laparotomy was performed on 13 patients $(40.62 \%)$ who had received lavage-peritoneal drainage. The average length of hospitalization was 4.18 days [95\% CI: 2.828491-4.981032 ]. There were two deaths (6.25\%). The most frequent reasons for consultation were: abdominal pain (43.75\%), perianal pain $(18.75 \%)$ and inguinal pain $(15.63 \%)$ (Table I).

Table 1. Reason for consultation

\begin{tabular}{|l|c|c|}
\hline Reasons for consultation & Headcount $(\mathrm{n})$ & Rate (\%) \\
\hline Abdominal pain & 14 & 43,75 \\
\hline Material and gas stop & 3 & 9.38 \\
\hline vomiting & 1 & 3.13 \\
\hline Abdominal wound & 3 & 9.38 \\
\hline Inguinal pain & 5 & 15,63 \\
\hline Perianal pain & 6 & 18.75 \\
\hline Total & 32 & 100 \\
\hline
\end{tabular}

The pathologies operated on in emergency were acute appendicitis $n=6(18.75 \%)$ and acute intestinal occlusions $n=6(18.75 \%)$ which are distributed according to the following etiology: by a sigmoid volvulus $n=1(3.13 \%)$, by a flange $n=1(3.13 \%)$, by a hail volvulus $n=1(3.13 \%)$ and by a sigmoid tumour in occlusion $n=3(9.38 \%$ ) (Table II).

Table 2. Distribution according to surgical pathologies

\begin{tabular}{|l|c|c|}
\hline Surgical Pathology & Headcount (n) & Rate (\%) \\
\hline Acute intestinal occlusion & 6 & 18.75 \\
\hline Penetrating wound of the abdomen & 3 & 9.38 \\
\hline Generalized acute peritonitis & 3 & 9.38 \\
\hline Acute appendicitis & 6 & 18,75 \\
\hline Strangulated inguinal hernia & 5 & 15,63 \\
\hline Liver contusion & 1 & 3.13 \\
\hline Acute lithiasic cholecystitis & 2 & 6.25 \\
\hline Perianal abscess & 3 & 9.38 \\
\hline Hemorrhoidal thrombosis & 3 & 9.38 \\
\hline Total & 32 & 100 \\
\hline
\end{tabular}

Appendectomy $n=6(18.75 \%)$ and hernia repair $n=5(15.63 \%)$ were the most common surgical procedures performed during the study. Surgical drainage of perianal abscesses $n=3(14.29 \%)$, and hemorrhoidectomy $n=3$ (14.29\%) were also performed (Table III). 
Table 3. Répartition selon les actes opératoires

\begin{tabular}{|l|c|c|}
\hline Operating acts & Headcount (n) & Rate (\%) \\
\hline Drainage wash only & 2 & 6.25 \\
\hline Eleorraphy & 2 & 6.25 \\
\hline Adhesiolysis & 1 & 3,13 \\
\hline Appendectomy & 6 & 18,75 \\
\hline Gastrorraphy & 2 & 6,25 \\
\hline Manual detorsion of the sigmoid & 1 & 3,13 \\
\hline Hernia treatment & 5 & 15,63 \\
\hline Sigmoidectomy + ileostomy & 2 & 6,25 \\
\hline Cholecystectomy & 2 & 6,25 \\
\hline Flattening an abscess & 3 & 9,38 \\
\hline hemorrhoidectomy & 3 & 9,38 \\
\hline Ileostomy and bowel resection & 1 & 3,13 \\
\hline ileostomy & 1 & 3,13 \\
\hline Intestinal anastomosis resection & 1 & 3,13 \\
\hline Total & 32 & 100 \\
\hline
\end{tabular}

The visceral emergency is the prerogative of the young subject whose average age is around 34.87 years old. Our study is similar to that of Berthe (2011) in Mali, who is 32.67 years old.

A sex ratio of 1.33 in favor of the male gender. Samoura (2011) in Mali reported a sex ratio of 1.66 in favor of the male sex. The following parameters: the age and sex of patients operated on for digestive surgery emergencies had not changed before or during the pandemic (Ai et al, 2020).

In Europe, they found a drop in the number of patients hospitalized for surgical emergencies during the pandemic, i.e. 59.3\% of the number in 2019, in contrast to our study, where an increase in the number of hospitalizations in our department was to be deplored because the other surgical centers were intended to treat only patients with Covid-19, therefore all surgical pathologies converge in the visceral surgery department (Luong-Nguyen et al, 2020). Visceral emergencies represented a quarter of the patients hospitalized in our department during the Covid-19 pandemic and they were all operated on in emergency. Despite the existence of the pandemic many patients came to the emergency department for visceral pathologies. This proves the permanent work of the doctors in the surgery department during the pandemic and we have seen the increase in the number of patients operated in emergency.

However, not all of these patients had had a coronavirus test at entry despite the presence of digestive signs related to the Covid-19 symptom. What increased

the risk of contamination for caregivers in front of an asymptomatic patient. Luong-Nguyen et al (2020) describe that systematic screening on admission of patients to surgical departments should be implemented by March 30, 2020. This routine screening was limited by the incompletely known incubation period and the low sensitivity of the PCR test, of the order of $60 \%$, which does not formally rule out the diagnosis of viral infection (Gornet et al, 2020).

An SARS-CoV-2 infection in a surgical inpatient involved both an individual and a collective risk. During this epidemic period, preventive measures were put in place to minimize the risk of 
contamination within hospital departments. These measures included increased training of health care staff, increased use of hydro-alcoholic solution and repeated hand washing, as well as the systematic wearing of surgical masks. At the same time, visits were restricted and then banned (Wong et al, 2020). Abdominal pain (43.75\%) was the most frequent reason for visiting the visceral emergency department. It had revealed intra-abdominal affections that translate into pain, often the location of the pain signs the affected organ according to the quadrant of the abdomen. The intensity of the pain pushes people to consult an emergency room, which is often hyperalgesic. Abdominal pain was the most common sign of digestive surgery emergencies (Jin et al, 2020). Some digestive manifestations of Covid19 were represented by abdominal pain, vomiting and diarrhea, which coincided with the signs of digestive emergencies (Soumah et al, 2020). This increased the risk of delayed management by waiting for a screening result for Cov- 2 infection in one specialist department before transferring to another surgical department (Collard et al, 2020). The pathology operated on in the emergency department was represented by acute intestinal occlusion (18.75\%) with the following etiology: a sigmoid volvulus, a volvulus of the graft, a sigmoid tumor, and a secondary flange. Occlusion is an unpredictable condition, its presence has nothing to do with the Covid pandemic19. It was one of the most common causes of exploratory laparotomy in surgery. The typical picture was a combination of vomiting, material and gas arrest, abdominal pain and abdominal meteorism (Slim \& Veziant, 2020). Xrays of the abdomen without preparation showed confirmation of the presence of hydroaeric levels. The abdominal CT scan was very sensitive and specific but was not available to all patients.

Some authors advocate the non-surgical treatment of digestive disorders such as uncomplicated appendicitis, cooling of acute cholecystitis, in order to support the effort against the progression of the Covid-19 epidemic and prefer to minimize the use of the operating room (Patriti et al, 2020). However, this decision favours the transition to the complicated form of these conditions, which are normally operated on in an emergency (Gorter et al, 2020). Non-surgical treatment should only be considered in cases where it is indicated according to evidence-based recommendations.

It should be noted that some proctological conditions had been operated on as emergencies, such as hemorrhoidal crises (9.38\%) and perianal abscesses (9.38\%). Hemorrhoids are a condition linked to several factors such as sedentary lifestyle. Sporting activity is a protective factor, they are not allowed to go out in public during the confinement period and they tend to forget to practice a sport activity.

The operating procedures vary according to the pathology being treated. An exploratory laparotomy was performed in (40.62\%). Exploration allows the etiology of the acute abdomen to be detected and treated. The specificity of the procedure depends on its etiology (Patriti et al, 2020).

An appendectomy is performed in the patient who has come in for acute appendicitis. It must be performed as soon as possible, within 12 hours of admission to hospital, to avoid the patient's transition from simple to complicated appendicitis (Slim \& Veziant, 2020).

After all surgical interventions, draconian protective measures were taken in accordance with national and local recommendations (Gorter et al, 2020). Postoperatively, the patient was admitted to a hospitalization sector adapted to his respiratory condition. During the epidemic 
period, the hospitalization areas were arranged as single rooms in order to limit cross-infections with other patients or family members.

Single rooms also facilitated isolation management. Visits were prohibited. Since this prohibition was intended to protect the patient, the visitor and staff (Slim \& Veziant, 2020). The average length of hospital stay is 4.18 days, which is close to that of Dick in Scotland (4.84 days) (Luong-Nguyen et al, 2020). The duration was the shortest in order to avoid the saturation of our service which is the only one available to treat surgical pathologies in our city. But at the same time, this avoids the risk of patients contracting a nosocomial SARS-CoV-2 infection (Wong et al, 2020).

\section{Conclusion}

Digestive ailments were present during the lockdown period and the coronavirus pandemic. It requires urgent surgical procedures. An increase in the number of patients hospitalized in the surgical department was visible during the pandemic. A heavy task for visceral surgeons since their activities were uninterrupted. All of this requires the establishment of an important measure of protection against the risk of contamination of health workers.

\section{References}

Ai, T., Yang, Z., Hou, H., Zhan, C., Chen, C., Lv, W., ... \& Xia, L. (2020). Correlation of chest CT and RT-PCR testing in coronavirus disease 2019 (COVID-19) in China: a report of 1014 cases. Radiology, 200642.

Berthé, I. (2008). Prise en charge des urgences chirurgicales digestives dans le service de chirurgie «A»du CHU du Point G (Doctoral dissertation, Thèse Med, Bamako, Université de Bamako, 2009, N09M53).

Collard, M., Lefevre, J. H., \& Maggiori, L. (2020). Antibiotics alone as an alternative to appendectomy for uncomplicated acute appendicitis in adults: changes in treatment modalities related to the COVID-19 health crisis. Journal of Visceral Surgery.

Gornet, J. M., Minh, M. L. T., Leleu, F., \& Hassid, D. (2020). What do surgeons need to know about the digestive disorders and paraclinical abnormalities induced by COVID-19?. Journal of Visceral Surgery.

Gorter, R. R., Eker, H. H., Gorter-Stam, M. A., Abis, G. S., Acharya, A., Ankersmit, M., ... \& Bruntink, M. (2016). Diagnosis and management of acute appendicitis. EAES consensus development conference 2015. Surgical endoscopy, 30(11), 4668-4690.

Jin, J. M., Bai, P., He, W., Wu, F., Liu, X. F., Han, D. M., .. \& Yang, J. K. (2020). Gender differences in patients with COVID-19: Focus on severity and mortality. Frontiers in Public Health, 8, 152.

Luong-Nguyen, M., Hermand, H., Abdalla, S., Cabrit, N., Hobeika, C., Brouquet, A., ... \& Sauvanet, A. (2020). Nosocomial infection with SARS-Cov-2 within departments of digestive surgery. Journal of visceral surgery, 157(3), S13-S18.

Patriti, A., Eugeni, E., \& Guerra, F. (2020). What happened to surgical emergencies in the era of COVID-19 outbreak? Considerations of surgeons working in an Italian COVID-19 red zone. Updates in Surgery, 1-2. 
Samoura, L. (2011). Prise en charge des urgences chirurgicales digestives au CSRéf de Bougouni.

Slim, K., \& Veziant, J. (2020). Urgent digestive surgery, a collateral victim of the Covid-19 crisis?. Journal of Visceral Surgery.

Soumah, S. A., Ba, P. A., Diallo-Owono, F. K., \& Toure, C. T. (2001). Les abdomens aigus chirurgicaux en milieu africain: étude d'une série de 88 cas à l'hô-pital Saint Jean de Dieu de Thiès. Sénégal Surgical acute abdominal emergencies in an African area: study of 88 cases at Saint Jean de Dieu hospital in Thiès. Senegal. Arch Surg, 136(5), 556562.

Wong, S. H., Lui, R. N., \& Sung, J. J. (2020). Covid-19 and the digestive system. Journal of gastroenterology and hepatology, 35(5), 744-748. 